Mots. Les langages du politique

\title{
Visions du temps. Cioran analyste de la réaction, de l'utopie et du progrès
}

Space and movement to represent time. Cioran'criticism about reactionary thought, utopia and progress

Visión del tiempo. Cioran analista de la reacción, de la utopía y del progreso

Jean-Claude Guerrini

\section{(2) OpenEdition}

Journals

Édition électronique

URL : https://journals.openedition.org/mots/6093

DOI : $10.4000 /$ mots.6093

ISSN : 1960-6001

Éditeur

ENS Éditions

\section{Édition imprimée}

Date de publication : 1 mars 2002

Pagination : 27-43

ISBN : 2-84788-007-0

ISSN : 0243-6450

\section{Référence électronique}

Jean-Claude Guerrini, « Visions du temps. Cioran analyste de la réaction, de l'utopie et du progrès ». Mots. Les langages du politique [En ligne], 68 | 2002, mis en ligne le 29 avril 2008, consulté le 23 avril 2022. URL : http://journals.openedition.org/mots/6093 ; DOl : https://doi.org/10.4000/mots.6093 


\title{
Visions du temps Cioran analyste de la réaction, de l'utopie et du progrès
}

\author{
« Nos conceptions politiques nous sont dictées \\ par notre sentiment ou notre vision du temps ». \\ Cioran, Joseph de Maistre, Essai sur la pensée \\ réactionnaire ${ }^{1}$. \\ « C'est toujours le temps qui glisse vers l'es- \\ pace, comme si le langage était mieux propre /.../ à \\ saisir la réalité spatiale que toute autre ». \\ Paul Zumthor, La mesure du monde ${ }^{2}$. \\ «Les idées sont aussi des affects. En particulier \\ l'acceptation du changement et la foi dans l'avenir \\ sont des dispositions de sentiment autant que des \\ pensées ». \\ Paul Bénichou, Le temps des prophètes ${ }^{3}$.
}

Parmi les repères symboliques qui permettent à des adversaires politiques ou idéologiques d'identifier leurs amis et leurs ennemis, ceux que propose la dimension du temps revêtent, au moins depuis les Lumières, une importance décisive. Dès lors que les agents historiques prétendent procéder à une rénovation, à une restauration ou inaugurer un cours nouveau, ils sont amenés à établir des hiérarchies temporelles.

\footnotetext{
- Groupe Rhêma, Université Lumière Lyon 2, 5 avenue Pierre Mendès-France, CP 11, 69676 Bron Cedex.

1. E.M. Cioran, Euvres, Exercices d'admiration, Essais et portraits, Quarto Gallimard, 1995, p. 1534. Abréviations utilisées : C : Sur les cimes du désespoir, 1934; L : Le livre des leurres, 1936 ; B : Bréviaire des vaincus : écrit entre 1941 et 1944, publié en 1993 ; P : Précis de décomposition, 1949 ; S : Syllogismes de l'amertume, 1952; H : Histoire et utopie, 1960 ; Ch : La chute dans le temps, 1964 ; I : De l'inconvénient d'être né, 1973 ; Éc : Écartèlement, p. 1979 ; Ex : Exercices d'admiration, Essais et portraits, 1986.

2. P. Zumthor, La mesure du monde, Le Seuil, 1993, p. 27.

3. P. Bénichou, Le temps des prophètes, Gallimard, 1995, p. 117.
} 
Les désignations, les qualifications et les évaluations que suscite la prise en compte de faits révolus et de projets, fatalement inaccessibles à l'expérience directe, appellent une médiation qui les mette en perspective et les inscrive dans un ordre temporel. C'est ce que permet un transfert métaphorique dont le point, la ligne, la courbe, le mouvement fournissent le support cognitif (essor, chute, déclin, progrès, révolution, retour, etc.), produisant ainsi un espace de représentation ${ }^{4}$ et une axiologie. Pour peu que l'échelle adoptée conduise à embrasser la totalité de la trajectoire historique, on pourra parler, avec K. Pomian, de « chronosophie $»^{5}$.

L'œuvre de Cioran présente à cet égard un intérêt particulier. Critique incisif des idéologies, encensé pour l'acuité de ses aphorismes et la fulgurance de ses sentences, l'écrivain roumain est tout le contraire d'un esprit dispersé. Par acharnement critique, par souci de se placer au vif des enjeux, il répertorie et passe au crible les principaux choix idéologiques qui s'offrent au jugement. Il se trouve ainsi amené à comparer les « visions du temps » qui leur correspondent, en se montrant particulièrement sensible à l'imaginaire spatio-dynamique qui s'y investit. Car il est indubitable que Cioran présente certaines dispositions favorables à la « tentation chronosophique ». Si celle-ci suppose l'identification, sur le modèle augustinien, de l'histoire humaine à une histoire individuelle, l'écrivain ne cesse, quant à lui, de penser sa vie dans l'horizon de cette analogie. Il établit, dès ses écrits de jeunesse marqués par l'hyperbole lyrique, un lien étroit, jamais démenti, entre sa destinée et le cours entier de l'histoire du monde : « Je ne peux vivre qu'au commencement ou à la fin du monde » (C. p. 81).

Ne cessant de méditer sur la mort et le sens de la vie, mais aussi sur l'histoire, il appréhende cette dernière à travers une image globale jalonnée par ses grandes époques ou ses évènements cruciaux, mythiques ou réels (Le Paradis, La Chute, les quatre âges d'Hésiode, les grandes civilisations, la décadence des empires, la quête de l'absolu ou de l'utopie, l'Apocalypse). Indifférent aux détails, il raisonne et juge à partir d'une véritable épure temporelle, le statut de la révolution, de la réaction, de l'utopie ou du progrès étant examiné en référence aux évalua-

4. « Le temps entre donc tout naturellement dans un système de représentation qui, au lieu d'être tridimensionnel comme celui que nous construisons pour l'espace, n'a qu'une dimension, le point et la ligne. Ainsi, s'il est dit que nous usons de métaphores spatiales pour parler du temps, il ne peut s'agir que de métaphores faisant appel à des termes unidimensionnels, qui n'impliquent ni des surfaces, ni des volumes » (A. Borillo, « Le déroulement temporel et sa représentation spatiale en français », dans Syntaxe et figuration du monde, Cahiers de praxématique, 27, Praxiling, Université de Montpellier, 1996, p. 109).

5. K. Pomian, L'ordre du temps, Bibliothèque des histoires, Gallimard, 1984. 
tions que peuvent susciter positions, directions, mouvements et rythmes à l'échelle de la trajectoire totale.

Notre étude cherchera à distinguer le rôle joué par les mots de l'espace et du mouvement dans les "visions du temps » analysées par Cioran. Elle débutera par l'examen des commentaires que lui a suggérés, dans Joseph de Maistre, Essai sur la pensée réactionnaire, l'œuvre d'un auteur-parangon qu'il cite abondamment. Elle puisera ensuite ses exemples dans les écrits français de la période 1949-1979.

\section{Réaction et vision statique du monde}

Conscient, au lendemain de la guerre, de figurer parmi les vaincus ${ }^{6}$, dégrisé de ses délires et de ses enthousiasmes, Cioran n'entend pas pour autant sombrer dans l'inconsistance et le ressentiment. Sa critique de la doxa progressiste et révolutionnaire, qui s'adresse à un auditoire non averti de son parcours, se présente comme un prolongement de la dénonciation du fanatisme qui l'avait conduit, avec ses amis de l'avantgarde roumaine (Mircea Eliade, Constantin Noïca notamment), dans l'impasse fasciste. Dans cette perspective, l'analyse de la pensée réactionnaire, malgré les différences qu'elle présente avec le vitalisme moderniste revendiqué par les émules de la Grande Roumanie, constitue une propédeutique stimulante.

En décidant, dans les années 1950, de consacrer une étude à Joseph de Maistre en préface à une réédition de divers textes parmi lesquels $D u$ Pape, pamphlet outrageusement traditionaliste et théocratique, Cioran choisit à coup sûr la provocation. Ce texte, essentiel pour la compréhension de son œuvre, sera repris sous le titre d'Essai sur la pensée réactionnaire aux éditions Fata Morgana en 1977 et assurera l'ouverture d'Exercices d'admiration en 1986. Ce qui intéresse Cioran chez ce pamphlétaire monarchiste et papiste, ce n'est évidemment pas sa défense des hiérarchies de l'ancien régime ou sa fureur ultramontaine, mais sa

6. Sur le passé fasciste du jeune Cioran, cette mise au point parait indispensable: «Pas l'ombre d'un penchant réactionnaire, en effet, chez le jeune Cioran, comme le voudrait un contresens couramment commis /.../ Son discours se caractérise au contraire par un credo ultramoderniste, un acquiescement à l'urbanisation, au monde et à la technique qui le situent quelque part entre les nationaux-bolcheviks ou le jeune Jünger, bien plus que dans l'orbite de la droite conservatrice et orthodoxiste roumaine de l'entre-deux guerres ", A. Laignel-Lavastine, « De l'inconvénient d'avoir été fasciste », Le débat, 93, janvier-février, p. 102-103. Voir aussi P. Bollon, Cioran l'hérétique, Gallimard, 1997, p. 54 à 121 ; N. Parfait, Cioran ou le défi de l'être, Desjonquères, 2001, p. 75 à 100. 
radicalité. Maistre fait la théorie d'un pouvoir absolument antidémocratique, dans lequel certains, comme Isaiah Berlin, ont vu une préfiguration du totalitarisme' ${ }^{7}$. Il fournit ainsi à l'analyste, ou à l'ancien fanatique à l'heure des bilans, l'occasion de se porter au cœur d'une logique politique.

Le sous-titre choisi, Essai sur la pensée réactionnaire, appelle d'emblée une remarque. Les oppositions lexicales jouant dans la définition des camps politiques un rôle crucial, l'emploi, à partir de 1794, du mot réaction puis la création sur le moule de révolutionnaire de l'antonyme réactionnaire servent à désigner l'attitude consistant à contrarier ou à inverser le cours de la Révolution. Ces mots seront au centre de l'interdiscours politique induit par l'interminable évaluation de l'évènement inaugural ${ }^{8}$. Or, dès son apparition, réaction perdant, dans la plupart des cas, le sens neutre qu'il gardait dans le couple action/réaction de la mécanique newtonienne, se voit chargé d'une valeur intrinsèquement dépréciative. Ceci vaut plus encore pour réactionnaire qui évoque l'agent d'une action ne s'exerçant que de manière négative pour entraver un processus jugé émancipateur. L'Essai devrait donc être lu, si l'on suit l'orientation argumentative induite par ce sous-titre, comme un réquisitoire. Et, de fait, cette présentation de l'œuvre d'un inconditionnel de l'absolutisme s'achève sur la nécessité d'une apologie de l'hérésie. Mais, et c'est ce qui en fait tout l'intérêt, cet « exercice d'admiration » - ne l'oublions pas -, est d'une écriture prodigieusement empathique et polyphonique et laisse s'exprimer largement, outre les voix fréquemment consonantes du moraliste ou du « spectateur » désabusé, le point de vue de l'« adversaire ».

Tout concourt, dans cet essai de 40 pages, à faire, au moins en apparence, de l'opposition Réaction/Révolution, une opposition structurante. La récurrence forte des lexèmes formant l'opposition ( 7 pour réaction, 13 pour réactionnaire, 30 pour révolution, 12 pour révolutionnaire), l'usage des majuscules théâtralisant le conflit, l'emploi de l'adjectif substantivé construisant un type (le réactionnaire, le révolutionnaire) installent le lecteur dans une disposition dualiste; les réactionnaires sont définis avant tout contre la Révolution (conservateur et tradition

7. I. Berlin, Le bois tordu de l'humanité, Romantisme, nationalisme et totalitarisme, Bibliothèque Albin Michel, Idées, 1992, p. 100-174.

8. «Est-ce une pure coïncidence si l'émergence du couple lexical révolution/réaction est à peu près contemporaine de la polarisation de l'espace politique ? Aristocrates et sans-culottes, clergé réfractaire et clergé assermenté, côté droit et côté gauche de l'Assemblée, partout s'établissent des oppositions. Dans le langage de l'époque, la "révolution" eut d'abord pour opposé la "contre-révolution". Celle-ci se doubla de la "réaction" » (J. Starobinski, Action et réaction, Vie et aventures d'un couple, Le Seuil, 1999). 
servant parfois de parasynonymes) ; le camp adverse est évoqué à travers une série de lexèmes plus variés qui esquissent une brève histoire de l'héritage de la Révolution : libéral, libéralisme (dans l'acception qu'ils avaient en France au $19^{\mathrm{e}}$ siècle), utopie, utopique, gauche, homme de gauche, démocrates, démocratiques, anarchiste, Progrès (ou progrès), les fervents de l'avenir. L'ensemble met en scène deux paires lexicales présentées, au centre du texte, comme indispensables à l'exercice du jugement politique, révolution/réaction et droite/gauche:

« Si fragile que soit au reste la distinction classique entre le concept de révolution et celui de réaction, nous devons néanmoins la conserver... Elle constitue un point de repère aussi problématique qu'indispensable » (Ex. p. 1546).

" "Droite" et "gauche", simples approximations dont malheureusement on ne peut se passer » (ibid.).

Pourtant tout le travail critique de Cioran va consister à mettre en crise cette vision dichotomique, très rassurante dans sa simplicité, à travers l'examen des « visions du temps » qui correspondent aux deux camps en présence.

Pour rendre compte de la «chronosophie» du « réactionnaire», Cioran, se référant à la pensée de Maistre, met en évidence la recherche d'une cohérence qui repose en profondeur sur des schèmes spatiaux apparemment hétérogènes, l'organisation temporelle de l'histoire et son rapport à la transcendance conduisant à privilégier certaines modalités spatiales et à en suspecter d'autres.

C'est ainsi que chez Maistre, l'optimisme des Lumières, en tant que refus obstiné de prendre acte de la Chute, constitue un désordre assimilable à une « crise du site » :

« Il n'y a que violence dans l'univers ; mais nous sommes gâtés par la philosophie moderne, qui dit que tout est bien, tandis que le mal a tout souillé, et que dans un sens très vrai, tout est mal, puisque rien n'est à sa place » (cité dans Ex. p. 1522).

La réponse à cette aggravation paroxystique de l'état du monde qu'est la Révolution, est une vision de l'histoire clairement ordonnée, dans la mesure où la perfection et l'ordre ont un lieu indiscutable, pleinement identifié : l'origine. Maistre, en un geste métaphysique dont Cioran raille l'ambition grandiose, renverse en effet l'ordre de succession qui faisait surgir la Révolution au terme d'un processus civilisateur, et situe curieusement la civilisation en amont du cours historique :

«Voilà la civilisation placée avant l'histoire ! Cette idolâtrie des commencements, du paradis déjà réalisé, cette hantise des origines est la marque même de la pensée "réactionnaire", ou, si l'on préfère "traditionnelle"» (Ex. p. 1532). 
L'auteur des Soirées de Saint-Pétersbourg souhaite en effet retrouver les secrets de la «science primitive» dispensés jadis par des "êtres d'un ordre supérieur » (cité dans Ex p. 1532). Vision de l'histoire que Cioran est amené naturellement à décrire en termes de "retour» (Ex. p. 1537) :

« L'histoire, suivant Maistre, doit nous faire revenir - par le détour du mal et du péché à l'unité de l'âge paradisiaque » (Ex. p. 1532).

Il s'agit d'échapper aux effets d'une déchéance seconde, mais néanmoins située dans un passé primordial, qui ne peut que se répéter et s'approfondir. L'invariabilité de la nature humaine ne laissant espérer aucun salut ici-bas, malgré la nostalgie d'un passé révolu, le « réactionnaire » perçoit l'histoire comme essentiellement vaine, comme le « cadre où se déroule le processus monotone de notre dégradation » (Ex. p. 1533). D'où, une valorisation forcenée de l'immobilisme :

« Toujours le réactionnaire, ce conservateur qui a jeté le masque, empruntera aux sagesses ce qu'elles ont de pire, et de plus profond : la conception de l'irréparable, la vision statique du monde » (Ex. p. 1533).

Son seul espoir est de voir l'histoire se clore (Ex. p. 1541), grâce à la survenue de la fin des temps, de l'Apocalypse. Selon Cioran, cette phobie du parcours résulte d'une suspicion visant au fond tout mouvement, notre séparation d'avec le tout constituant le premier partage spatial, cause de tout mal.

Pour mieux faire ressortir le rôle joué dans la pensée de Maistre par cette extériorité radicale du fondement de la légitimité justifiant une politique de l'autorité installée dans la permanence, Cioran cite d'ailleurs une image surprenante, où l'écrivain réactionnaire, désireux de disqualifier la Révolution et inspiré par l'imaginaire mécanique du $18^{\mathrm{e}}$ siècle, compare l'attitude des Français à celle d'enfants trop avides, mus par une curiosité maladroite :

« Ils ont voulu voir dedans : ils ont mis à découvert les principes politiques, ils ont ouvert l'œil de la foule sur des objets qu'elle ne s'était jamais avisée d'examiner, sans réfléchir qu'il y a des choses qu'on détruit en les montrant » (cité dans Ex. p. 1535).

\section{L'illusion du but : l'utopie et le paradis}

Face au « réactionnaire », le « révolutionnaire » refuse de se voir enchainé à un monde «d'avant l'histoire » et fait le pari d'une fécondité 
$\mathrm{du}$ « déroulement historique » (Ex. p.1534). Cioran souligne cette fois l'association de la croyance en la perfectibilité de l'être humain et de la confiance dans le temps :

« Autrement généreuse, car autrement naïve, la pensée révolutionnaire, elle, associant à l'effilochement du devenir l'idée de substantialité, discerne dans la succession un principe d'enrichissement, une féconde dislocation de l'identité et de la monotonie, et comme une perfectibilité jamais démentie, toujours en marche» (Ex. p. 1534).

C'est là, sans que Cioran le mentionne, restituer une corrélation (perfectibilité/toujours en marche) présente en effet dans le texte inaugural de Benjamin Constant, Des réactions politiques (1797), où le penseur libéral, au moment même où il introduit le mot réaction dans le langage politique, mobilise, après Condorcet, la métaphore de la «marche en avant » de l'esprit humain :

« Depuis que l'esprit de l'homme marche en avant /.../ il n'est plus d'invasion de barbares, plus de coalition d'oppresseurs, plus d'évocation de préjugés, qui puisse le faire rétrograder. /... / Il faut que les lumières s'étendent, que l'espèce humaine s'égalise et s'élève, et que chacune de ces générations successives que la mort engloutit, laisse du moins une trace brillante qui marque la route de la vérité ${ }^{9}$.

Cette croyance dans le mouvement en avant et vers le haut (s'élève), cette idée d'une marche irréversible sont à l'opposé du diagnostic réactionnaire, dégagé par Cioran, d'une dégradation irréparable et d'une aspiration à un « retour».

Après l'échec de la Révolution (pour autant qu'elle prétendait procéder sur le champ à une rénovation radicale de l'humanité), les utopistes ont senti que la mobilisation des énergies impliquait un changement dans la perception du cours historique. C'est pourquoi Cioran, dans Histoire et utopie, donne une importance majeure au geste théologicopolitique, symétrique de celui qu'il a attribué à Maistre, consistant à transformer la référence au paradis, autrefois ancrée dans le passé, en orientation progressiste :

« Incapables de le [le paradis] déceler dans le tréfonds de leur nature, trop pressés aussi pour pouvoir l'en extraire, les modernes devaient le projeter dans le futur, et c'est un raccourci de toutes leurs illusions que l'épigraphe du journal saint-simonien Le Producteur: "L'âge d'or, qu'une aveugle tradition a placé dans le passé, est devant nous"» (H., p. 1053).

Quand il ne s'abandonne pas à la pure et simple dérision («Fermé depuis cinq mille ans, le paradis fut rouvert, selon Jean Chrysostome, au moment où le Christ expirait. /.../ Tout porte à croire qu'il est de nou-

9. B. Constant, Écrits et discours, éd. O. Pozzo di Borgo, Jean-Jacques Pauvert, 1964, vol. 1, p. 127. 
veau verrouillé et qu'il le restera longtemps encore »H. p. 1040) ou qu'il ne se contente pas de rappeler l'étymologie (« Utopie rappelons-le signifie nulle part» H. p. 1037), Cioran s'efforce de mettre en évidence l'illusion spatiale qui inspire la pensée utopique, notamment ce paradoxe directionnel qui l'incite à situer au terme de l'histoire la nostalgie de son début :

«Si elle [la nostalgie] retourne à la source du temps, c'est pour y retrouver le paradis véritable, objet de nos regrets. Tout à l'opposé, celle dont procède le paradis d'ici-bas sera démunie de la dimension du regret précisément : nostalgie renversée, faussée et viciée, tendue vers le futur, obnubilée par le "progrès", réplique temporelle, métamorphose grimaçante du paradis originel » (H. p. 1041).

À cette torsion spatio-temporelle (renversée, faussée, tendue vers le futur) s'ajoute un paradoxe : en contradiction avec le dynamisme de l'ouverture au possible qu'elle revendique, l'utopie, qui prétend, dans un avenir proche, donner effectivement accès au paradis, est vouée à figer le devenir, à engendrer une « durée stationnaire, de Possible immobilisé » (H. p. 1052), « un monde statique » (H. p.1048), promis à une « stagnation totale » (H. p. 1036).

\section{La marche du progrès}

Entre les pôles du paradis regretté, situé dans un au-delà ou un endeçà, et celui du paradis espéré ici-bas, l'espace du parcours subit à son tour l'épreuve de la critique. On a vu comment dans Des réactions politiques de Benjamin Constant, la métaphore de la marche en avant était étroitement liée à l'idée de perfectibilité et à l'impossibilité de toute rétrogradation. S'inscrivant dans une tradition d'évolution sémantique du mot progrès (Pascal, Leibniz, Turgot Condorcet), cette figure devait constituer l'un des soutiens symboliques les plus puissants de la modernité, sous la plume de nombreux écrivains et théoriciens (dont le comte de Saint-Simon, Auguste Comte, Victor Hugo, Pierre Leroux, Proudhon, etc. $)^{10}$. C'est que le mot marche à travers ses emplois directionnel et non-directionnel, constitue une véritable aubaine rhétorique : capable de valoriser une volonté enthousiaste (le déplacement en avant d'un être vertical tourné dans un certaine direction), mais aussi le mou-

10. Deux ouvrages de P.-A. Taguieff apportent, outre de précieuses analyses, de nombreuses citations qui constituent une sorte d'anthologie : L'effacement de l'avenir, Galilée, 2000 ; Du progrès, Librio, 2001. 
vement régulier et contrôlé (l'enchainement réglé des pas), ce polysème permet tout aussi bien de figurer l'épanouissement heureux d'une activité résultant du premier apprentissage de l'autonomie que le bon fonctionnement d'un mécanisme. Image prototypique de la maitrise, la marche en avant est donc éminemment apte à incarner une modernité conquérante, nécessaire, assurée de sa légitimité ${ }^{11}$.

Sans doute Cioran ne soumet-il à aucun moment cette métaphore à une "déconstruction " systématique. Mais on peut affirmer qu'elle hante littéralement son œuvre. Son écriture, par la récurrence des marques lexicales et des allusions, crée un intertexte interne qui corrode impitoyablement les valorisations optimistes et mobilisatrices d'une métaphore devenue rapidement stéréotype ${ }^{12}$.

La croyance en la perfectibilité de l'homme corrélée à un schème cinétique nécessaire et irrépressible est ainsi présentée comme un leurre. L'histoire, selon Cioran, est le déploiement passif d'un dérisoire cortège d'impostures, une pure successivité :

«L'Histoire n'est qu'un défilé de faux absolus, une succession de temples élevés à des prétextes, un avilissement de l'esprit devant l'improbable» (P. p. 581).

«L'histoire se déroule sans plus, indépendamment d'une direction déterminée, d'un but » (H. p. 1043).

Toutes les tentatives pour modifier l'homme en fonction d'un but se soldent d'ailleurs par des échecs qui vont à contresens de l'intention initiale ou qui faussent son intention de départ. L'histoire atteint ses buts à l'envers :

« On réalise l'opposé de ce qu'on a poursuivi, on avance à l'encontre du beau mensonge qu'on s'est proposé /.../ Sur chaque être pèse la menace de rétrograder vers son point de départ (comme pour illustrer l'inutilité de son parcours et l'inutilité de tout parcours) » (Éc., p. 1413).

À l'actant-sujet présupposé par les discours optimistes (l'Humanité) peut donc se substituer une allégorie disqualifiante :

11. Voir J. Picoche, "La définition des polysèmes, ses bases, ses ressources, ses limites ", exemple du mot marcher, dans Études de lexicologie et de dialectologie, Conseil international de la langue française, 1995, ainsi que M.-L. Honeste, " De l'empreinte au mouvement », dans Corps en mouvement, édit. A. Vaillant, Publications de l'Université de Saint-Etienne, 1996.

12. «Les données discursives sont souvent indirectes ou implicites, éparses ou lacunaires. Dans la mesure où il ne se fonde pas sur la répétition littérale, le stéréotype doit souvent être recomposé à partir d'éléments diversifiés qu'il faut ramener au trait typique ", R. Amossy, L'argumentation dans le discours, Discours politique, Littérature d'idées, Nathan Université, 2000 , p. 111. Ce qui est dit ici pour la formulation du stéréotype vaut aussi pour sa critique. 
« L'Histoire est l'ironie en marche, le ricanement de l'Esprit à travers les hommes et les évènements » (P. p. 708).

« L'histoire, paradis des somnambules, obnubilation en marche » (Éc. p. 1410).

Car l'homme ne maitrise pas sa marche. Les modalités de son mouvement (somnambulique, précipité, erratique) sont à l'opposé de ce que suggère le prototype harmonieux rêvé par les prophètes d'une coordination heureuse des trajectoires de la nature, de l'homme, du beau, du bien, du vrai...

«Au degré de mobilité que nous avons atteint, nous ne sommes plus maitres de nos gestes et de notre sort » (Ch. p. 1094).

«Nous avançons en masse vers une confusion sans analogue, nous nous dresserons les uns contre les autres /.../ plus personne ne daignera vivre, si ce n'est pour liquider et se liquider» (Éc. p. 1431).

D'où l'emploi de termes dépréciatifs pour désigner un mouvement dépourvu de sens qui peut s'interpréter comme un signe de « décomposition » : automatisme, bougeotte (Ch. p. 1091). La vitesse, forme de dissipation de l'espace/temps, érigée en critère du degré de civilisation, emporte les existences individuelles et les sociétés dans un activisme, une compétition perpétuels et obligatoires : quiconque, simple illettré ou peuple rétrograde, prétendra y échapper devra être ramené dans le droit chemin (Ch. p. 1095). Loin d'être une manifestation de la liberté et d'une recherche de la perfection, le prétendu sens de l'histoire impose son emprise tyrannique :

« Des restes d'humanité, on n'en trouve encore que chez les peuples qui distancés par l'histoire, ne mettent aucune hâte à la rattraper. À l'arrière-garde des nations, nullement effleurés par la tentation du projet, ils cultivent leurs vertus démodées, ils se font un devoir de dater. "Rétrogrades", ils le sont assurément, et persévèreraient volontiers dans leur stagnation, s'ils avaient les moyens de s'y maintenir» (Ch. p. 1087).

L'obsession du mouvement, cette « désertion en avant », (Éc. p. 1430), cet « élan suprême vers le pire » (C. p. 1094) dont le dynamisme et l'accélération volatilisent le but et la direction, ne résulte pas d'une soumission à la technique. Pour Cioran, celle-ci est la conséquence, non la cause du phénomène. Pire encore, le progrès, en dépit du stéréotype de la marche, est incapable de préserver un contact avec le sol du concret :

« La plus grande des folies est de croire que nous marchons sur du solide. Dès que l'histoire se signale, nous nous persuadons du contraire. Nos pas paraissaient adhérer au sol, et nous découvrons brusquement qu'il n'y a rien qui ressemble au sol, qu'il n'y a rien non plus qui ressemble à des pas » (Éc. p. 1451). 
L'ignorance de la direction et du sens de la marche, la perte de contact avec le sol de la réalité, n'empêchent pas pour autant la compétition acharnée et la prétention à assumer la conduite du mouvement :

« Nous en voulons à tous ceux qui ont choisi de vivre à la même époque que nous, qui courent à nos côtés, gênent nos pas ou nous laissent en arrière » (H. p. 1025).

« Tous veulent diriger les pas de tous » (P. p. 584).

On aurait tort, remarquons-le, de réduire cette critique à un simple propos sociologique. Car elle répond en profondeur, après plusieurs décennies, aux enthousiasmes du jeune Cioran dont le lyrisme se grisait du dynamisme frénétique d'une génération intellectuelle tiraillée entre prophétisme mystique et réaction apocalyptique :

« Ceux qui ignorent les feux ardents de l'esprit prophétique accueillent la succession des instants dans leur relativité et, sceptiques, acceptent tout. Ce n'est que dans la prophétie qui enjambe le temps, que nous vivons l'instant en regard de la direction absolue, vers laquelle il faudrait tendre. Les fins dernières, la prophétie nous les rend accessibles dans le vécu exacerbé du moment » (L. p. 163).

« Marche arrière vers le chaos originel, retour à la confusion primordiale, au chaos originel ! Élançons-nous vers le tourbillon antérieur à l'apparition des formes. Que nos pensées palpitent de cet effort, de cette démence, de cette flambée, de ces gouffres !» (C. p. 80$)$.

Mais désormais l'humour remplace le lyrisme. Cioran joue avec les clichés et inverse radicalement l'axiologie habituellement associée à l'image de la marche : tout mouvement est déprécié, tout sémantisme suspect stigmatisé, quitte à réveiller une métaphore endormie, une catachrèse, ou à réactiver une étymologie.

«Un grand pas en avant fut fait le jour où les hommes comprirent que, pour pouvoir mieux se tourmenter les uns les autres, il leur fallait se rassembler, s'organiser en société » (H., p. 1043).

« L'homme ne bouge que pour faire le mal» (H., p. 1013).

«L'homme est parti du mauvais pied» (I. p. 1377).

« Tout projet est une forme camouflée d'esclavage» (Éc. p. 1499).

C'est donc tout naturellement, que sur un registre plus grave, Cioran en vient à reformuler la Chute en termes horizontaux et à énoncer un appel à la démobilisation (vivre à contre-courant, saboter la marche) :

«Tout pas en avant, toute forme de dynamisme comporte quelque chose de satanique : le "progrès" est l'équivalent moderne de la Chute, la version profane de la damnation /.../ que sommes-nous sinon des réprouvés en marche, prédestinés à l'immonde, à ces 
machines, à ces villes, dont seul un désastre exhaustif pourrait nous débarrasser » (Ch. p. 1087).

« Est vice tout ce qui s'ajoute à nos impératifs profonds /.../ Est vertu en revanche tout ce qui nous induit à vivre à contre-courant de notre civilisation, tout ce qui nous invite à en compromettre et en saboter la marche » (Ch. p. 1090).

Faut-il en conclure que l'Essai de 1957 dissimulait en réalité une profonde adhésion à la pensée de Maistre et à son idéal "statique »? Cioran ne cessait en effet, dans cet « exercice d'admiration », dont il est impossible, dans les limites de cet article, de décrire l'extrême habileté polyphonique, d'incriminer la corruption engendrée par le mouvement et le temps, et développait dans ses digressions narquoises, un humour dévastateur, peu compatible avec son adhésion de façade à la doxa démocratique :

«Assoupis, nous le sommes tous ; et, paradoxalement, c'est pour cela que nous agissons. Continuons donc comme si de rien n'était, pratiquons nos distinctions traditionnelles ${ }^{13}$, heureux d'ignorer que les valeurs surgies dans le temps sont, en dernière instance, interchangeables » (Ex. p. 1547).

Mais son scepticisme le situait désormais à mille lieues des certitudes de Maistre, et on aurait tort de ne pas prendre au sérieux le parti pris d'hérésie qu'il opposait à son absolutisme, parti pris que viendra confirmer, plus de dix ans plus tard, cette phrase des Cahiers (p. 585) d'une tonalité inhabituelle : « La seule valeur à laquelle je crois est la liberté ». L'éloge amusé, dans le Précis de décomposition, des « Anges réactionnaires », plus avisés que le bouillant Lucifer, « blottis sous leurs ailes sans âge, éternellement vainqueurs et vaincus en Dieu, insensibles aux néfastes curiosités » (P. p. 615), est sans doute plus proche encore de la vérité ; comme aussi cette boutade d'Écartèlement : « Seriez-vous réac? - Si vous voulez, mais dans le sens où Dieu l'est » (Éc. p. 1462) ${ }^{14}$.

13. Réaction/révolution, droite/gauche.

14. Devant cette défiance systématique à l'égard des illusions du mouvement et de la « marche en avant », on est en tout cas légitimement conduit à se demander si on ne se trouve pas devant une formulation spatiale des concepts-clés de la «rhétorique réactionnaire » tels que les identifiera A. O. Hirschman : inanité, effets pervers, mise en péril (A. O. Hirschman, Deux siècles de rhétorique réactionnaire, Fayard, 1991 (trad. P. Andler), [The Rhetoric of Reaction : Perversity, Futility, Jeopardy, The President and Fellows of Harvard College, 1991]). 


\section{Cioran, le temps et l'expérience de la « mauvaise éternité »}

À ce désir de retrouver l'état de pure virtualité, antérieur à toute temporalité et tout mouvement, un drame personnel, l'insomnie, souvent évoqué par l'auteur, tant dans ses œuvres que dans ses Cahiers ou dans les entretiens de la fin de sa vie, fournit un éclairage utile. Il suggère un lien étroit, revendiqué, entre affect et pensée, dont surgit une nouvelle « vision du temps » singulière, marginale, mais censée préfigurer le destin futur d'une humanité libérée, peut-être à ses dépens, du fardeau des oppositions idéologiques.

Face au temps affronté dans la monotonie des nuits, l'écrivain, convaincu de parvenir à une forme exceptionnelle de lucidité, a pu mesurer la vanité des conflits des dormeurs et cultiver le sentiment d'une différence et d'une supériorité de veilleur, vécue dans une tension explosive :

«Lorsque pèse sur vous le poids de l'éternité, lorsque vous entendez le tic-tac d'une horloge ou le battement des secondes, comment ne pas ressentir l'inanité de la progression dans le temps et le non sens du devenir ? À quoi bon aller plus loin, à quoi bon continuer ? /.../ Lorsque cette révélation se produit la nuit, l'absurdité des heures qui passent se double d'une sensation de solitude anéantissante, car - à l'écart du monde et des hommes - vous vous retrouvez seul face au temps, dans un irréductible rapport de dualité » (C. p. 101).

Dans un premier temps, on l'a vu, la réponse enthousiaste et prophétique d'un messianisme fasciste était venue combler l'angoisse du non sens en postulant la possibilité d'un bond hors du temps :

« N'aimons-nous pas les prophètes parce qu'ils annulent le temps ? La prophétie est un saut de la conscience hors du temps » (L. p. 162).

Mais, après les déceptions du fanatisme, l'approfondissement de cette relation frontale au temps présenté comme un «faux jeton à l'échelle métaphysique » (Ch. p. 1154), sur fond de pessimisme radical (formulé dans les termes mêmes de Maistre : « Dans ce monde rien n'est à sa place, en commençant par ce monde même» P. p. 615), conduisit Cioran à un approfondissement de sa critique.

Associant l'acceptation du temps et de son rythme trompeusement régénérant à une forme de passivité et de médiocrité, il présente la corruption de l'action dans le temps comme la Chute elle-même, ce qui l'amène dans l'ouvrage de 1964, intitulé précisément La chute dans le 
temps, à déborder fréquemment la représentation courante du temps comme ligne, et à libérer ainsi la virtualité d'une extériorité :

«Après avoir gâché l'éternité vraie, l'homme est tombé dans le temps, où il a réussi sinon à prospérer, du moins à vivre : ce qui est certain, c'est qu'il s'en est accommodé. Le processus de cette chute et de cet accommodement a nom Histoire » (Ch. p. 1156).

Constatant son incapacité à être "porté » et " protégé » par les instants comme les autres hommes (Ch. p. 1152), Cioran aurait pu se réjouir de se trouver ainsi à l'écart de l'agitation générale. Or, loin d'accéder à l'éternité originelle qu'il appelait de ses vœux, il prend progressivement conscience d'une nouvelle chute, d'une déchéance aggravée. Distinguant dorénavant deux types de «chute », il formule une curieuse topologie temporelle qui repose sur l'opposition prépositionnelle $d a n s / d u$ et finit par réhabiliter le temps considéré comme enveloppe protectrice, comme « élément vital» :

«Les autres tombent dans le temps ; je suis moi tombé du temps. À l'éternité qui s'érigeait au-dessus de lui succède cette autre qui se place au-dessous, donc stérile, où l'on n'éprouve plus qu'un seul désir : réintégrer le temps, s'y élever coute que coute, s'en approprier une parcelle pour s'y installer, pour se donner l'illusion d'un chez-soi. Mais le temps est clos, mais le temps est hors d'atteinte : et c'est de l'impossibilité d'y pénétrer qu'est faite cette éternité négative, cette mauvaise éternité » (Ch. p. 1152).

Cette déchéance temporelle ne saurait être comprise, au demeurant, indépendamment de l'expérience de la solitude à laquelle Cioran l'associe continument. L'écrivain émigré, viscéralement allergique, depuis l'embardée fusionnelle de sa jeunesse roumaine, à toute forme d'engagement ou de participation à la comédie sociale, revendiquait, certes, une indépendance absolue. D'une vigilance constante, malgré la chaleur de sa conversation et sa jovialité partout attestées, Cioran était décidé à ne dépendre de personne et proclamait hautement son aspiration à ce qu'on serait tenté de nommer un statut d'《 atopie ».

« Une patrie, c’est de la glu » (Éc. p. 1456).

« Je m'intéresse à n'importe qui sauf aux autres. J'aurais pu être tout sauf législateur » (Ch. p. 1474).

« Jusqu'à présent j'étais étranger dans tous les sens du mot, c'est-à-dire un non citoyen. J'ai cessé de jouer un rôle quelconque. Dans l'être, je ne suis qu'un ci-devant » (Cahiers p. 969, 2 février 1972).

« N'avoir jamais l'occasion de prendre position, de se décider ni de se définir, il n'est vœu que je forme plus souvent» (P. p. 991). 
Pourtant, ayant dépassé une sorte de point d'équilibre, Cioran finit par ressentir cette chute du temps comme une rupture de trop avec les autres, qui l'installe désormais, dans un néant social non voulu :

« Tant que nous demeurons à l'intérieur du temps, nous avons des semblables, avec lesquels nous entendons rivaliser; dès que nous cessons d'y être, tout ce qu'ils font et tout ce qu'ils peuvent penser de nous, ne nous importe plus guère » (Ch. p. 1156).

Cette expérience d'une chute au second degré aurait pu, tout au plus, constituer une anomalie personnelle. Mais Cioran, cédant sans doute à un besoin irrépressible de prophétiser, y vit le signe avant-coureur d'une phase nouvelle de l'humanité, marquée par la dissipation des illusions engendrées par la croyance dans le temps :

« Mais voici que menace une autre chute /.../ Cette fois-ci il ne s'agira plus de tomber de l'éternité, mais du temps ; et, tomber du temps, c'est tomber de l'histoire, c'est, le devenir suspendu, s'enliser dans l'inerte et le morne, dans l'absolu de la stagnation, où le verbe lui-même s'enlise, faute de pouvoir se hisser au blasphème ou à l'imploration. Imminente ou non cette chute est possible, voire inévitable » (Ch. p. 1156).

«Avoir perdu et l'éternité et le temps ! L'ennui est la rumination de cette double perte. Autant dire l'état normal, le mode de sentir officiel d'une humanité enfin éjectée de l'histoire » (Ch. p. 1157).

L'émergence d'une post-histoire apparait donc comme la généralisation d'un processus vécu et exploré malgré lui par l'auteur : la destruction du moi dans la solitude conjuguée à une érosion de l'élément médiateur qui rendait possible l'énoncé même des différends, à savoir la référence à une temporalité collective, dont on a vu combien les figures spatiales auxquelles elle se prêtait permettait l'expression de points de vue opposés.

Ultime conséquence de cette dissipation de la perspective historique : la tolérance suscite des sentiments ambivalents chez ce « libéral intraitable » (Ch. p. 983) qui, bien que refusant de prendre position, envisageait avec circonspection l'extinction des illusions contradictoires dont il avait fait sa cible :

« L'Histoire confirme le scepticisme ; cependant elle n'est et ne vit qu'en le piétinant ; aucun évènement ne surgit du doute, mais toutes les considérations sur les évènements y conduisent et le justifient. C'est dire que la tolérance - bien suprême de la terre - en est en même temps le mal. Admettre tous les points de vue, les croyances les plus disparates, les opinions les plus contradictoires, présuppose un état général de lassitude et de stérilité. On en arrive à ce miracle : les adversaires coexistent - mais précisément parce qu'ils ne peuvent plus l'être ; les doctrines opposées se reconnaissent des mérites les unes aux autres parce qu'aucune n'a de vigueur pour s'affirmer » (P. p. 728). 


$$
\text { * } *
$$

Réaction, utopie, marche du progrès, chute dans le temps, chute du temps... On trouve donc chez Cioran une sorte de cartographie des impasses auxquelles, dans l'état actuel de notre imaginaire temporel, seraient confrontés ceux qui prétendent dessiner la figure de l'avenir ou indiquer la voie opportune. Que cette topique idéologique recoure si massivement à des oppositions spatiales et dynamiques s'explique à la fois par la prégnance effective de ce type de figures dans les représentations temporelles et par une disposition particulière de l'écrivain roumain. C'est ce qu'avait fort bien repéré Maurice Nadeau, dès la parution du Précis de décomposition, quand il mettait la pensée de Cioran sous le signe du chemin :

« Au bord de la voie triomphale qui mène à l'âge atomique, il jette le sac et s'assied, fourbu, refusant toutes les consolations et toutes les pitiés $\gg{ }^{15}$.

Aujourd'hui, l'angoisse ne surgit plus seulement de la menace d'un cataclysme nucléaire, mais de la dissipation du fantôme de la voie triomphale.

On peut cependant proposer de cette crise de l'avenir, une interprétation dédramatisée. Dans un article figurant dans l'ouvrage collectif intitulé « Peut-on encore croire au progrès? », le philosophe J.-M. Besnier affirme ainsi :

«Ce sont peut-être seulement les justifications que l'on donne à l'action dans le monde et dans l'Histoire qui sont sur le point de changer. Ce sont peut-être seulement les figures rhétoriques qui soutenaient la dynamique des efforts individuels et collectifs qui sont en passe de se modifier $\gg{ }^{16}$.

Certains jugeront ce diagnostic exagérément serein. Il montre en tous cas que l'écriture de Cioran, sa sensibilité panoramique aux « visions du temps » ancrée dans une expérience des investissements émotifs qu'elles présupposent, allaient au cœur d'une conjoncture idéologique dont il aura contribué à dépouiller la scénographie.

15. M. Nadeau, «Un penseur crépusculaire », Combat, 29 septembre 1949.

16. J.-M. Besnier, «La volonté d'avenir en défaut», dans D. Bourg, J.-M. Besnier (dir.), Peut-on encore croire à l'idée de progrès ?, PUF, 2000, p. 41. 